\title{
Conversation on a common manifesto
}

\section{Alvado Bañón, Joaquín1; Hadjisoteriou, Maria; Devereux, Mike ${ }^{3}$}

${ }^{1}$ Alicante University, Spain joaquin.alvado4@gmail.com

${ }^{2}$ University of Nicosia, Cyprus hadjisoteriou.m@unic.ac.cy

${ }^{3}$ UWE Bristol, UK

mike.devereux@uwe.ac.uk

Citation: Alvado Bañón, J.; Hadjisoteriou, M.; Devereux, M. (2021). "Conversation on a Common Manifesto." UOU scientific journal \#01, 26-33.

ISSN: 2697-1518. https://doi.org/10.14198/UOU.2021.1.04

This document is under a Creative Commons Attribution 4.0 International license (CC BY 4.0)

JA First, we need an Architectonical space for learning. We name it. Circus Island. 
JA Circus Island must be a constellation of public spheres as narratives of autonomous subjectivity. We will seek to enact the multidisciplinary direction through which architectonical practices and processes come most alive. We will constitute a map of contemporary knowledge circuits where art, theory, science, culture, ecology, and politics collides. Urban systems and metaterritory experience open to freedom openness and changes.

MD I think you have the right tone of excitement, exploration and creativity alongside a feeling of this being something new, for new times. What I feel is important with a manifesto is that it sets a framework in which to understand our adventure, to encourage contributions to a journal (and to judge them) and that this matches the aspirations of UoU architectural teaching. Amongst the vast range of journals and teaching experiences

we need to be identifiably different and communicate that. I like the analogy with 'constellation' as we are scattered around Europe and beyond with the UoU project - and just like a star in a constellation we are (I hope) illuminating architectural thinking and research to the maximum extent we can.

But I am a bit unclear here on your reference to 'public' when referring to spheres. UoU means - university of universities. A university (in the UK at least) for example is (apparently) a publically accessible institution, but access is controlled by the state education system and its examinations. You need these to achieve entry. The university itself is autonomous of the state (the public) and acts like a private institution (it costs students a minimum of $£ 9,250$ per year for their tuition) so that means that it is not public in the sense that l'd like architectural education to be. Many potentially good architects are excluded - the system

is public only in a limited way. I wonder if it is worth expanding upon 'public' to explain it more widely. In the case of the journal

I think 'public' should be used to encourage anyone with a voice to articulate an argument / research - not necessarily just academic (in the way you have invite student contributions).

JA UOU, our international studio, is an area of uncertainty and reflection. This space must be built among everyone, students, teachers, institutions, guests and citizens, hundreds of voices within a discursive dynamic system.

UOU, the "Circus Island" is a democratic space for discussion. 
MD I am very supportive of the multi-disciplinarity that you espouse to. Perhaps an explicit mention of 'technology' (unless you see it as 'science') might be something helpful to the reader, and perhaps alongside thoughts of 'urban systems' and 'metaterritory' it is worth balancing that with 'detailed' and 'micro' in terms of scale of investigation and discovery.

JA The construction of this experimental space need:

1. Openness. To incorporate open formats and new work contexts. Experimental practices and learning systems.

2. To incorporate student's upcoming and active vision. The student is part of our teaching. Our goal the autonomy based on their own maps of projective action.

3. To embrace diversity. Working on broad contexts. Students and teachers experience on their capabilities, knowledge and limits as an essential part for the teaching portfolio.

4 Proposal for the future. The space is for managing the future reality.

MH What is this future reality?

If we begin from the notion, as Lefevre argues, that (social) space is a (social) product, a dialogue on the potential role of the future architect is crucial in pedagogy.

Opening up vital questions as

'is the production of space a collaborative process?' 'can we make the process of architecture a dialogue?'

'how do we involve local actors- as co-authors?'

Allows for new definitions of the role that an architect has to play, going away from the idea of an architect as a dictating designer, but rather discuss it as a facilitator, an agent.

[Lefebvre H. The production of space. Blackwell; 1991.]

JA 5. Uncertainty. Emotion to work on the unknown to find the structure for the fiction. To accept complexity of knowledge in contemporary teaching.

6. Research as autonomy of thought. It is committed to scientific knowledge to expand research and knowledge.

7. Imagination. Imaginative space as a process of relationship.

8. To build an exhibition space, a document. UOU is a document, a portfolio itself.

9. Discussion as a search for knowledge. To accept the multidisciplinary approach of a changing profession.

11. Risk. The UOU was born in an unreferenced context, on a bizarre online periphery.

12. - Dynamicity. Travel for deferred knowledge. 
MD In terms of \#04 'the future reality' I wonder if we can be 'real' about the future. We, as designers, are I hope able to influence the future but I enjoy the experimentation and creativity that we bring away from our position away from reality. We might inform reality perhaps, but without being playful I would argue that our 'reality' is stifled and loses its potential. In some ways \#05 embraces what I am trying to say. Another point that springs to mind in reading the principles, and

I do not wish be pedantic here. You use the word 'scientific' and indeed it is in the title of the journal, but I am sure that you do not propose by this to demote the humanities and arts as disciplines and methods for and of research, or indeed as

means of teaching. Perhaps that needs drawing out more.

You do of course start off with this as a statement at the top of the manifesto so I am sure it is in your mind as an intended direction.

JA The Circus Island, the space for UOU, is a collective, dynamic, experiential and future process. It is a living and to live in. It is productive and exciting. It is an adventure, a risk.

MD You capture the important aspects of UoU: imagination, discussion, democracy, diversity etc... I would be inclined to think that we might emphasise a spirit of community (a 'common'). By this I mean that staff and students are mutually supportive of each other. The whole (of $\mathrm{UoU}$ ) is bigger than the sum of its parts. We are creating a space in which architectural education and research is able to grow by the help each participant (experienced or not) brings to it.

\begin{abstract}
JA Circus Island: A leisure space, a space for fun, to enjoy and to spend a good time, a space for happiness; a soft space, a space to love, to fraternize, to become close friends; a conflictive space, a space to fight, to discuss and to win.
\end{abstract}

MD Whilst I agree with the mood of your manifesto, and the way in which you are structuring it there is one phrase on which

I would urge caution; that is, "a space to fight, to discuss and

to win." Personally, I do not have to win. I enjoy learning and discussing, but winning is not my goal- educating, learning and enjoyment are my goals. For me, in UoU there is no 'winning' or 'losing.' If you are a participant (within the full meaning of that word - not just an attendee) then you cannot lose. 


\begin{abstract}
JA 1. Mask
"Architecture is a fuzzy amalgamation of ancient knowledge and contemporary practice, an awkward way to look at the world and an inadequate medium to operate on it... Architecture is too slow. Yet, the word "architecture" is still pronounced with certain reverence (outside of the profession). It embodies the lingering hope -or the vague memory of hope- that shape, form, coherence could be imposed on the violent surf of information that washes over us daily. Maybe, architecture doesn't have to be stupid after all. Liberated from the obligation to construct, it can become a way of thinking about anything -a discipline that represents relationships, proportions, connections, effects, and the diagram of everything."

Rem Koolhass. "Content"

Circus Island is a critical and experimental vision of contemporary urban society. We propose a Mask, an architecture about the making of architecture.

Masking ourselves as a process in which an individual changes or "masks" their natural personality. We will work to find our architectonical mask and build it. We will become architects As Hejduk in Victims, We will project our Island to portray an analogous city an experimental version of the contemporary city. Radicality, detour, digression as a critical reflection.

Architectural typology, to mobilize a repertoire of building elements drawn into an exchange between theatrical and formal/expressive practices.
\end{abstract}

MD I find your quote from Koolhaas appropriate. For me, he has tried hard to redefine architectural thinking. Not so much always in a purely, rational philosophical way but certainly intelligently. As he puts it "I think one of the important evolutions is that we no longer feel compulsively the need to argue, or to justify things on a kind of rational level. We are much more willing to admit

that certain things are completely instinctive and others are really intellectual." For us, I would argue that there is a place for the instinctive (and obviously the intellectual) and the 'mask' helps this. It allows us to suggest, to proclaim, to assert ideas that we feel, without always the need to be able to instantly justify. Through discussion and experiment we can test the ideas, but we can come to the 'island' without having done the testing. The methods of testing are innumerable, and you draw our attention to some important ones: The mask allows us to test without fear of judgment. If I am correct, Hedjuk draws us to the idea of a 'masque' (Berlin Masque) and not a 'mask'. The former is a form of entertainment (cp masquerade), often allegorical; whilst the latter is simply a face covering 9as we have to wear in these pandemic days). Personally, I see UoU as a drama, a 


\section{JA 2. Language}

Language systems reside in individual minds, they have a separate existence and thus remain detached from their users. Rorty define an "ironist as someone who fulfills three conditions: (I) She has radical and continuing doubts about the final vocabulary she currently uses, because she has been impressed by other vocabularies, vocabularies taken as final by people or books she has encountered;

(2) she realizes that argument phrased in her present vocabulary can neither underwrite nor dissolve these doubts;

(3) Insofar as she philosophizes about her situation, she does not think that her vocabulary is closer to reality than others that it is in touch with a power not herself". Ironists describe themselves are subject to change, always aware of the contingency and fragility of their final vocabularies, and thus of their selves.

The opposite of irony is common sense.

UOU needs a new vocabulary to establish the rules of our architecture. Let us find a foolish act or idea.

MD I completely agree. We need a new language of architecture. It needs to embrace a way of thinking beyond the physical. In that it can share intellectual space with many other disciplines. It can be ironic (ie. Richard Rorty) and it can even be cynical if it helps make a point, but it should never be destructive, only constructive. Architectural language has all too often been aloof and corrosive. My workshop later this semester will focus on that. Perhaps here is a good moment to expand upon your sense of finding a 'foolish act or idea' so that the point

\section{JA 3. Dynamic Knowledge}

In Circus Island we try to find the language throw the vocabulary.

The question is:

What do we refer to when we talk about Architecture?

This is the question for our architectural research at the UOU. We want to talk from scratch about innovation, social behaviour, nature, growth, time, change, sustainability, weather, citizenship, atmosphere, water, adaptability, welfare and environment.

We use research as a technical tool to work on diversity, an open future, emotion, imagination and a complete portfolio to establish a dynamic process that will help us formulate concepts 
concerning architecture and life.

Dynamic knowledge is related to time, space and existence. The online procedures are real dynamic systems, chaotic descriptions. In dynamic systems, time and space are interdependent; there is no apparent center or order. In our research, we focus on dynamic grids of geometry, which create the shape of a structure, thus giving architectural design presence. As a Dynamic system, we work with Iteration and sensitivity as initial conditions. It is under those parameters that a system process becomes a structure. Our approach is related to two main items: emptiness and dynamic systems. Emptiness is related to iteration and recursion, while dynamic systems are related to sensitivity to initial conditions.

Emptiness as a "democratic space" is projected as a conflict between virtual and real space. It gives architecture a kind of depth that is not exhausted on a spatial scale, but works jointly with the virtual space to manifest its void as a possibility. It is a probability, and it is shown as a domain within the realm of possibility. It transcends architecture's perception as an "object" at the moment or stage that we become aware of its existence. Networks connecting spaces are instruments for understanding their structural functions. Diagrams and grid maps depend on the network' shape and quality. In our research, networks are based on cultural background. New policies for sustainable cultural thought will become the answer to future design questions and new identity systems.

MH Inclusive Architecture / Urban Commons Raising awareness for and claiming the right of the people to the city as another aspect of addressing the Urban Commons could introduce a new system / a new identity.

Addressing people's diverse needs; understanding of the user, not as a generic person but as a unique individual that has the right to co-inhabit, co-appropriate the city, could radically shift the direction and essence of architecture and the built environment.

Could we facilitate people to appropriate architecture? Allowing users to act as equal social actors, can influence the way spaces are shaped.

How can we think of the notion of Commons in architecture? How is it converted in a spatial quality?

If we consider space as a container where social realities unfold in as Stavrides argues, then an investigation on strategies of performative space systems can unfold opportunities for various inhabitation and appropriation scenarios thus enhancing opportunities of Common activities and appropriation taking place in the city.

[Stavrides S. Common spaces of urban emancipation. Manchester: Manchester University Press; 2019] 
MD Here, I think the key word that you hit upon is 'iteration.' If design, both in academic research and in practice, is anything it is iterative. A conversation with ourselves and between ourselves that plays out in time, and now plays in virtual space.

I am not entirely clear what you mean here by 'emptiness' as a condition of the approach. Are you thinking, we start with a blank

canvas, a tabula rasa and that the dynamic system forms from there - like (back to your metaphor of a constellation) a big bang

theory? And, as you suggest, such an emptiness is somehow democratic? Does it need rules (like the laws of physics) to keep

it under control or is the emptiness to be left to its own devises so we see where it leads us?

JA Our approach works with dynamic, online structures.

Each unit becomes a tensional network of visual negotiations.

Virtuality reinforces the effect of chaotic order in such a way that certain "informal" elements become structural supports for space. The visual complex of spatial interconnections is established through democratic architecture as an emptiness. The tensional organisation is based on grids, on creating threedimensional connections in which order is magnified into a labyrinth of structures at a small-object scale.

MD I think the 'dynamic online structures' is a helpful aidemémoire but the link between democratic and emptiness needs elaboration to be clearer to an audience not familiar with such terminology.

\begin{abstract}
JA Circus Island is a dynamic system. Dynamic systems' sensitivity to initial conditions means that a system's asymptotic behaviour can be altered by even the slightliest of changes in the initial conditions: "the butterfly effect". As we well know, we need to educate the next generations to stay true to the initial conditions.
\end{abstract}

MD What I understand by your 'dynamic system' is that it evolves but is always useful - it informs. You refer to this (as I read it) as creating 'new policies for sustainable cultural thought.' That is laudable.

Again, thanks very much for the thought provoking draft. It has certainly made me think and become even more enthusiastic (if it is possible to be even more enthusiastic!) about UoU. I would be very happy to discuss any of this further and look forward to 